\title{
Bangla Digital Number Plate Recognition using Template Matching for Higher Accuracy and Less Time Complexity
}

\author{
Mohammad Jaber Hossain \\ Student, Department of Computer \\ Science \& Engineering, American \\ International University- \\ Bangladesh
}

\author{
Md. Hasan Uzzaman \\ Student, Department of Computer \\ Science \& Engineering, American \\ International University- \\ Bangladesh
}

\author{
A. F. M. Saifuddin Saif \\ Assistant Professor, Department \\ of Computer Science \& \\ Engineering Bangladesh
}

\begin{abstract}
The importance of vehicle number plate detection is increasing day by day notably in Bangladesh for digitalized transportation system. Particularly in situation to find out the lost vehicle, improvement of security, access of vehicles in restricted areas, to save time in toll gates and detecting the guilty vehicles which are involved in road accidents or other related crime. This paper represents a method of Bangla number plate recognition. The propose method have four main steps-preprocessing, number plate extraction, segmentation of characters and character recognition. In preprocessing step need to convert the test image in gray level first and then binarized using Otsu method. Sobel edge operator, morphological dilation and erosion are used to find the plate location. Boundary box feature in used for segmentation of characters. Then template matching technique was applied for recognition of the characters. The proposed method is more effective to extract the license plate region. The recognition rate and time required to extract is also improved then some other studies done earlier.
\end{abstract}

\section{Keywords}

Number Plate detection, Template matching.

\section{INTRODUCTION}

In recent years, use of vehicles in Bangladesh is increasing rapidly due to the increase of population. Besides problem involved vehicles are increasing every day. Like robbery with vehicles, not maintaining traffic rules properly, reckless driving and unnecessary overtaking caused major accidents. Necessity of vehicle number plate detection is also increasing because of high density of vehicles in roads and increased crime related to vehicles. It helps to detect the guilty vehicles who are involved in the crime. Besides it can use in different parking areas to take records of cars and in toll booths for toll collection as well.

In Bangladesh there are different types of digital number plates, green background number plates are for commercial use, white are for personal use vehicles. Ref [1] works with commercial vehicle number plates where they extract number plates using color feature. All of the number plates are two lines in and have Bengali letters and numbers. First line indicates the area name and the type of the vehicle, second line represents the class of the vehicle and the vehicle registration number. In most of the previous works with other countries vehicle number plate have one line number plates.

In recent years there are a number of methods applied for Bangla vehicle number plate detection, but still there are many challenges to overcome. There are 4 main stages of VNPR which is preprocessing of captured image, detection of license plate region, segmentation of characters and finally recognition of segmented characters. In preprocessing, convert the given image in gray scale and then that gray scale image converted to binary image. For extraction sobel edge operator used to detect the edges and then applied morphological dilation \& erosion to localize the number plate. Boundary box feature is used to segmentize the characters. For recognition of characters template matching was applied which helped to train the templates of Bengali words, letters and numbers first, then match the templates with extracted characters of test image. Hough transform is used to detect vertical edges in [20] to find out the region of license plate. Morphology based license plate extraction is used in [21]. Ref. [2,3] used template matching for recognition in their previous work. Templates are resized into $24 * 42$ pixel images and background is converted into black, letters and characters converted into white. Then segmented characters are matched with templates.

The next part of the paper contains as follows, Research Background in section 2 which will discuss related number plate recognition in previous study, Proposed Research Methodology in section 3 which will contain proposed steps, Experimental Results and Discussion in section 4 and Conclusion in section 5 .

\section{RESEARCH BACKGROUND}

There are a lots of works on vehicle number plate detection in previous study. Ref. [4] converted the image in grayscale image in preprocessing step and applied dilation on that gray image. To detect the number plate image binarization is important in preprocessing, $[13,14]$ applied binarized image in their previous work .In Ref. [10] to deal with different light condition of evening and noon and to convert the intensity, binarization is applied by determine threshold. Otsu method is used for thresholding which determine the intensity of binary image in [16]. Dilation and erosion is used in preprocessing step in [15]. 
Ref. [8] used combined color information and template matching technique to get license plate location. They used a template of blue rectangle and were searching for vertical pixel by converting the image in HSV format. Ref. [7] applied different methods to localize the number plate, they used the local intensity change feature, the color feature and tag feature. Once license plate is recognized others are skipped to save the time. Ref. [2] used histogram based license plate localization to extract the license plate for Indian vehicle number plates. Sobel mask used in [9] to extract vertical edges and to get the candidate region of license plate histogram analysis was applied, then morphological operators is used to detect the Number plate.

Vertical and Horizontal projection used in [12], here vertical projection helps to determine the starting and ending of the line and horizontal projection use for segmentation. As Bangla vehicle number plates have more than one line, Ref. [5] used horizontal projection for line separation and then applied vertical projection for segmentation of the characters. Ref. [2] segmented letters and numbers based on connected components and specified a certain ratio of area for template matching to recognize the characters. Optical Character Recognition is applied in [11] to classify or recognize the letters and numbers of number plates and convert them to readable text into the computer. It's needed to remove boundaries in preprocessing of image to recognize the characters. Ref [17] used defocusing fuzzy logic and CNN for character recognition. Back propagation neural network proposed in $[18,19]$ for number plate recognition, but it requires more time to train, although recognition time after training is lower. Ref.[4] used feature vector for recognition, they makes a dataset of characters, train the feature of characters and classify.

\section{PROPOSSED METHOD}

In this Section, there will be the description of the methods and techniques. The system is designed for recognizing of Bangla vehicle license number plate. In general term, the model consists of mainly four computational steps. First one is pre-processing of the acquired image which is crucial to get better performance in further steps. Second, Number plate extraction by locating number plate location from the preprocessed image. Third, segmentize the characters using boundary box feature from the extracted plate. In the final step, Recognize the segmentize characters using Template matching. Details of the method is discussed below. In Fig. 1 flowchart of the proposed method is given.

\subsection{Image Acquisition:}

In the image acquisition step, images acquired from the roadside vehicle. Images was captured in the morning and evening sunlight from different distance, particularly in between 1-2 meters using the mobile camera with different resolutions. Image of license plates was captured from both front and rare side of the vehicles.

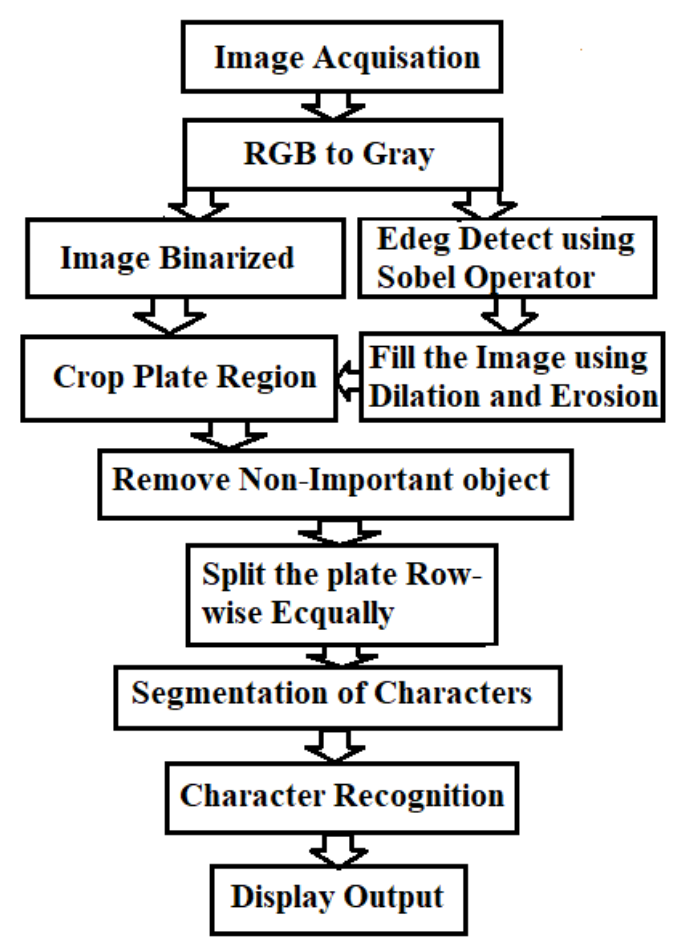

Fig 1: Proposed Model

\subsection{Pre-Processing:}

Pre-processing is essential to get better result of extraction and recognition. There is a sample of test image given in (Fig. 2). The image was converted to Grayscale from RGB image first. The RGB image is 24 bit and processing is timeconsuming. So need to convert the color image into the grayscale image (Fig. 3). The formula to convert the RGB image to gray value is:

$$
\text { Gray value }=0.3 * \mathrm{R}+0.59 * \mathrm{G}+0.11 * \mathrm{~B}
$$

A compact pattern representation is also defined in the preprocessing step. Binarization process converts the grayscale image into the binary image (Fig. 4) using the Otsu method by calculating the threshold $(\mathrm{T})$ value as [16]. $\mathrm{T}$ is calculated by Eq. 2 .

$$
g(x, y)=\left\{\begin{array}{l}
1 \text { if }(x, y) \geq T \\
0 \quad \text { otherwise }
\end{array}\right.
$$

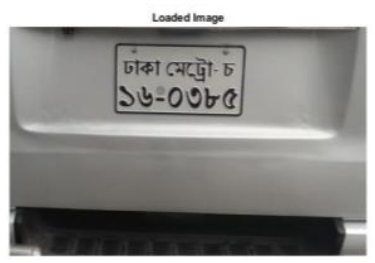

Fig 2: Test Image

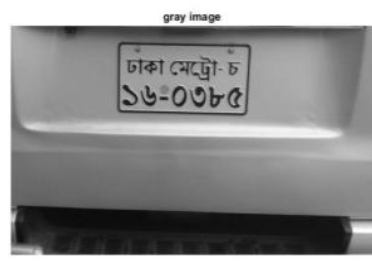

Fig 3: Gray Image 

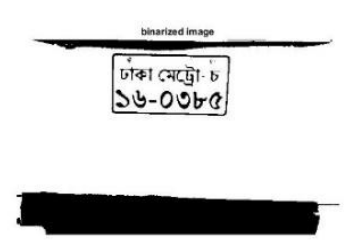

Fig 4: Binarized Image

\subsection{License Plate Extraction:}

The license plate extraction step is very important for this system. Firstly, need to localize the license plate area and then crop the license plate from the image. To localize the license plate first Sobel edge operator was used on the grayscale image to detect the edges in Fig. 5(a). After applying the Sobel operator morphological dilation and erosion were applied in Fig. 5(b). Morphological dilation \& erosion process image based on shapes. Dilation set on 0 on the binary image for assumed pixels and erosion sets 1 on the binary image for assumed pixels. From the output image license plate area was roughly localized. Then boundary feature, horizontal and vertical projection used to locate plate area. Then crop the plate area using binarized image. The cropped image is shown in Fig.6(a). Then need to clear the dust and remove unnecessary edges and borders (Fig. $6 b)$.

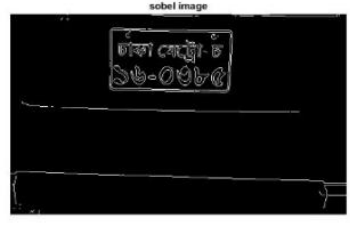

(a)

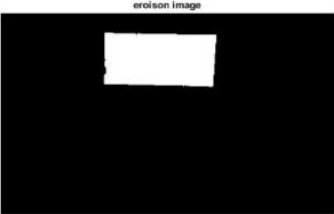

(b)
Fig 5: The left image shows Sobel applied on gray image and the right image shows fill image after dilation and erosion

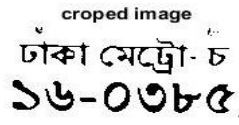

(a)

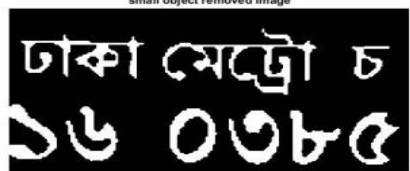

(b)
Fig 6: The left image is the extracted number plate image and the right one is dust removed image

\subsection{Character Segmentation:}

As Bangla vehicle number plates are double line number plates, firstly need to divide the number plate row-wise equally in two parts. Upper part (Fig. 7) carries the characters and the lower part (Fig .8) has the digits. Then segmentize sequentially upper part and the lower part from left to right. Boundary feature is used to segmentize the characters from the extracted number plate. First, need to get the number of connected objects in the plate portions. Then find the smallest rectangle having the region using bounding box property. Then extract every region from the plate portions and resized as [24 42] pixels. The similar process applied for the lower part of the number plate.

\section{जास्ता विद्धा ए}

Fig 7: Number plate Upper Portion

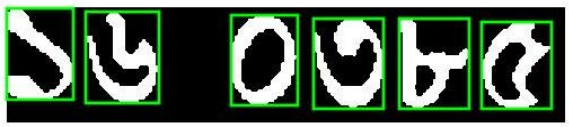

Fig 8: Number plate Lower Portion

\subsection{Character Recognition:}

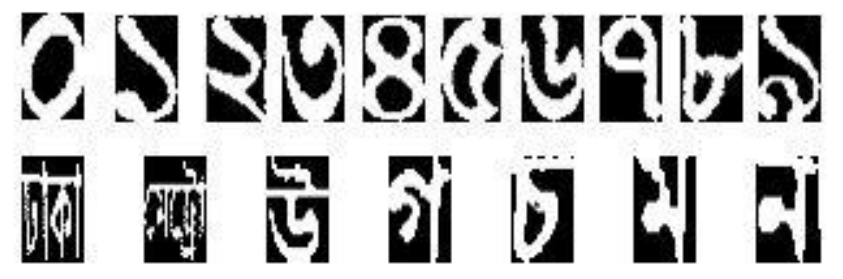

Fig 9: Sample Templates

Character recognition is the crucial part of vehicle number plate recognition. Template matching was applied here for the recognition, the size of the template was $24 * 42$ pixels with the black background and white color space characters (Fig. 9). For each character, measure the correlation coefficient $r$. Find out the best match between the extracted character and the template which is $\mathrm{d}$ and the template used for best matching with input letter is 1 .

$$
\begin{aligned}
& s=\mathrm{s}-\left(\sum_{t=1, j=1}^{m, n} s_{i, j} / m \times n\right) \\
& t=\mathrm{t}-\left(\sum_{t=1, j=1}^{m, n} t_{i, j} / m \times n\right) \\
& r \\
& =\sum_{t=1, j=1}^{m, n} t_{i, j} \times s_{i, j} / \sqrt{\text { 国 } \sum_{i=1, j=1}^{m, n} t_{i, j}^{2} \text { ? } \times \text { 田 } \sum_{i=1, j=1}^{m, n} s_{i, j}^{2} \text { ? }}
\end{aligned}
$$

Here, $\mathrm{t}$ is the template image and $\mathrm{s}$ is the segmentize image.

$$
d=\operatorname{argmax}(r), \boldsymbol{l}
$$

Took the result in a notepad (Fig.10) and to be more exact voice output also pronounced.

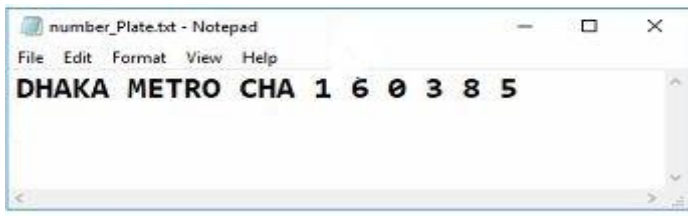

Fig 10: Output in Notepad 


\section{EXPERIMENTAL RESULTS \& DISCUSSION}

In this portion, brief idea will be given about experimental setup, datasets, experimental results, discussion and analysis of proposed method result and the result found in previous study.

\subsection{Experimental Setup}

For proposed method worked on the environment with configuration of Intel Core(TM) i5-8400 CPU, $2.80 \mathrm{GHz}$ processor with 4GB ram and 64bit Windows 10 operating System, worked in Matlab-2017a platform for simulation. Implement the method in Matlab where the toolbox was used to deal with different functions. It helps to process the image rigorously and designed as to work with different functions at a time besides it can process larger data within a short time. Matlab helps to visualize the work process nicely as well. For the experiment, pictures are captured from inside and outside parking areas which was image acquisition by the mobile camera which has a 13MP main camera with an $\mathrm{f} / 2.0$ aperture. The close up shoots are good to capture with this camera and the camera interface is clean and easy to use. Different type of vehicles number plate like cars, covered vans and trucks was considered to capture image. Other experimental steps of the method are done in Matlab. Here the image was captured manually and selected image manually to do the experiment. To improve the setup for real time application or in practical image can be captured automatically by camera and the proposed method will execute and the result will be in sheets which will records the number plates. For real time use some problem need to develop like number plate titled more than 10 degrees need to overcome and detection in different weather like rainy and sunny weather. Proposed method can perform on sunny weathers pictures only. The method can detect bordered number plates only, so borderless number plate cannot detect using the proposed method. Besides dirty number plates, blurry pictures, broken number plates and number plate with color missing in characters detection may not successful.

\subsection{Datasets}

For the experiment, had to look for a standard dataset by web surfing and from BRTA website. There are privacy information's in number plates, for that reason there may not any real time dataset from road transport authority or others. As there was no standard dataset to use, need to create own dataset by capturing good quality number plate pictures from the outside parking and parking inside the houses. Need to take permission to capture the image of the vehicles from the owner to make the dataset. Mobile camera was used to capture the images in morning and evening sunlight particularly in distance between 1 to 2 meters. The experimental result is increased by the quality of the pictures. In time of capturing pictures need to take good quality number plates which are not damaged, hand written or dirty number plates. Some of the number plates character colors are not visible anymore as number plate used for long time, sometimes a portion of characters are missing in number plates or a portion of number plate is broken or in some condition rectangular border of the number plates are not visible anymore. Besides sometimes number plates are hided because of the bumper or damage protection tools. So need to ignore those situations and number plates for the experimental data set. The test was performed on 50 pictures which are manually captured from road side parked vehicle, having front and rear view of number plates of different vehicles and all the number plates are two row type Bangla Digital Number plates. In this dataset includes two types background number plates, green background number plates are commercially used carry vans, trucks and the white color background number plates are cars and Hiaces which are personally used vehicle. All of the digital number plates are rectangular in shape. Characteristics of captured number plate given below:

Number plate type - Double line number plate Number plate background- Green, white Environment- Outside parking, inside parking Number Plate view- Front, Rear

Inage captured from Distance- $1 \mathrm{~m}$ to $2 \mathrm{~m}$

Detect plate Tilt up to -0 degree -10 degrees horizontally Number plate Shape- Rectangular

\subsection{Experimental Results}

Proposed method achieved significant success rate and less time complexity which is determined by experimenting the dataset.

Table 1: Result analysis

\begin{tabular}{|c|c|c|}
\hline Process & $\begin{array}{c}\text { Accuracy } \\
\text { according to } \\
\text { Number }\end{array}$ & $\begin{array}{c}\text { Accuracy } \\
\text { According to } \\
\text { Percentage }\end{array}$ \\
\hline $\begin{array}{c}\text { Number plate } \\
\text { Detection }\end{array}$ & $47 / 50$ & $94 \%$ \\
\hline $\begin{array}{c}\text { Character } \\
\text { Recognition }\end{array}$ & $45 / 47$ & $95.74 \%$ \\
\hline Overall Accuracy & $45 / 50$ & $90 \%$ \\
\hline
\end{tabular}

Table 2: Accuracy comparison with other methods

\begin{tabular}{|c|c|}
\hline Method & Overall Accuracy \\
\hline The Proposed Method & $90 \%$ \\
\hline$[1]$ & $88.8 \%$ \\
\hline$[2]$ & $80 \%$ \\
\hline$[4]$ & $84.80 \%$ \\
\hline$[7]$ & $81 \%$ \\
\hline
\end{tabular}

Table 3: Processing time of proposed method

\begin{tabular}{|l|l|}
\hline Process & Processing Time \\
\hline Detection & $0.1 \mathrm{sec}$ \\
\hline Segmentation & $0.044 \mathrm{sec}$ \\
\hline Recognition & $0.04 \mathrm{sec}$ \\
\hline Overall & 0.184 \\
\hline
\end{tabular}

Table 4: Processing time comparison with other methods

\begin{tabular}{|c|c|}
\hline Method & Processing Time \\
\hline The Proposed Method & $0.18 \mathrm{sec}($ average $)$ \\
\hline$[4]$ & $0.5 \mathrm{sec}$ \\
\hline
\end{tabular}




\begin{tabular}{|c|c|}
\hline$[22]$ & $0.284 \mathrm{sec}$ \\
\hline$[16]$ & $0.025 \mathrm{sec}$ \\
\hline$[5]$ & $1.2 \mathrm{sec}$ \\
\hline
\end{tabular}

Using the dataset, out of 50 pictures, 47 number plates extracted successfully which is $94 \%$ of test images, after applying template matching on that extracted pictures get $95.74 \%$ accuracy for character recognition and get $90 \%$ accuracy for overall method. The method took $0.1 \mathrm{sec}$ to extract the number plate, $0.044 \mathrm{sec}$ for segmentation and
$0.040 \mathrm{sec}$ to recognize the characters. The overall processing time of the method is $0.18 \mathrm{sec}$.

Table 1 shows the success rate of the method in different section of the experiment and Table 3 shows the time required to extract the number plate, segmentize the characters and finally to recognize the characters. The accuracy was is previous study and the proposed method for overall process shows in Table 2. Table 4 represents the processing time required in average of other methods and the proposed method. In Table 5 shows the test image, extracted number plate and result after recognition in notepad for both green and white color background number plates as sample.

Table 5: Sample test images and number plate extraction and recognition

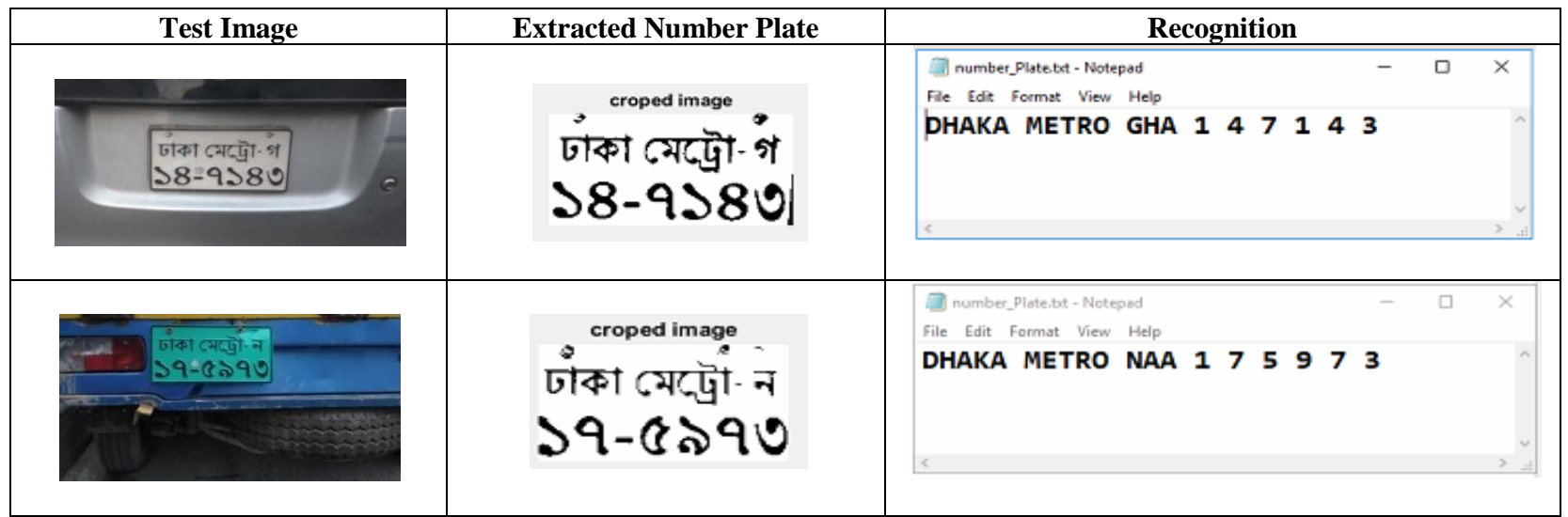

\subsection{Analysis and Discussion}

accuracy percentage of detection

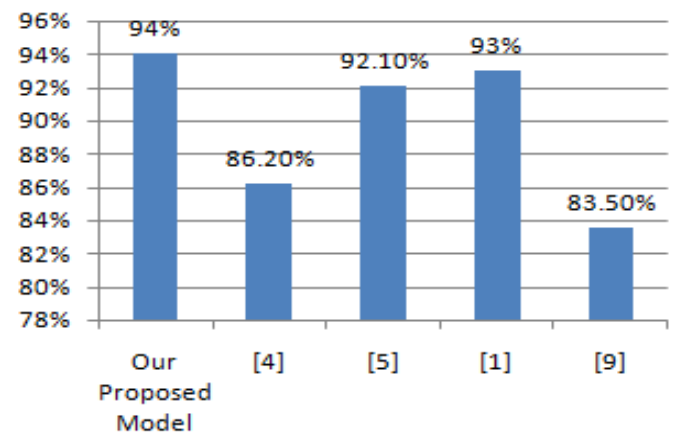

Fig 11: Accuracy comparison of number plate detection with some other methods

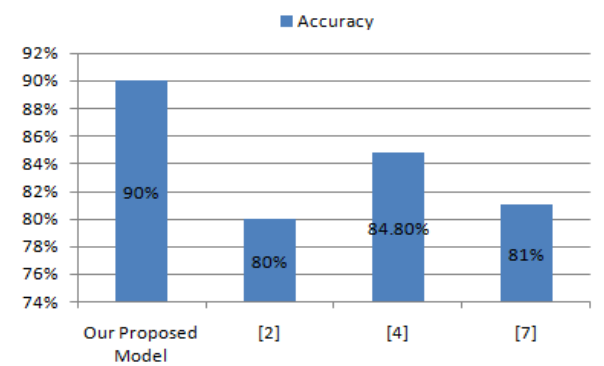

Fig 12: Accuracy comparison with some other previous works using template matching

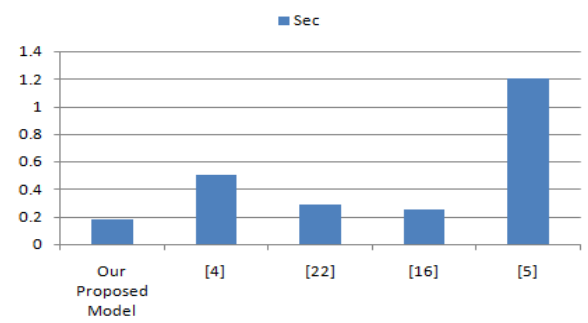

Fig 13: Processing time comparison with some other previous works

Fig. 11 shows the accuracy difference between some other method applied earlier and the proposed method for number plate detection, Fig. 12 shows the comparison of other methods and the proposed method overall performance of the number plate recognition methods where the proposed method get higher accuracy then others in detection and overall process as well. In Fig. 13 it shows the processing times of different methods where notably the proposed method took less time for overall recognition process.

Ref.[2] they used RGB to gray conversion and dilation first and then horizontal and vertical projection for localization, after segmentation they use template matching for 
recognition. Here they got $80 \%$ successful visible number plates.83.50\%accuracy found to localize number plate in [9] working with Iranian number plates. Ref [7] got $81 \%$ accuracy for overall process working on Egyptian number plates, they also followed template matching for recognition. $86.2 \%$ of successful extraction rate is found in [4] and $84.8 \%$ accuracy found in average on overall process. Ref [1] working on Bangla commercial number plates, get $93 \%$ detection rate and $88.8 \%$ recognition rate in overall experiment. Ref. [5] working with Bangla number plates as well get $92.1 \%$ accuracy for extraction and $84.16 \%$ accuracy for recognition. In the experiment 50 pictures was used, out of these pictures 47 number plates extracted successfully which is $94 \%$ of test images, from that extracted pictures got 95.74\% accuracy for character recognition and get $90 \%$ accuracy for overall method.

$0.25 \mathrm{sec}$ needed to extract the number plate in [4] where the proposed method needed $0.10 \mathrm{sec}$ to crop the number plate from the given image, $0.12 \mathrm{sec}$ required for segmentation but the proposed method took $0.044 \mathrm{sec}$ to segmentize the characters and the average time to recognize the characters of the method is $0.04 \mathrm{sec}$, but they required $0.1 \mathrm{sec}$. Ref. [22] needed $0.284 \mathrm{sec}$ for the total process. Besides in [16] they required $025 \mathrm{sec}$. Ref. [5] need $1.2 \mathrm{sec}$ for overall process working with Bangla number plates. But the proposed method took $0.18 \mathrm{sec}$ in average for overall process.

The experiment was on different size and resolution images. And the processing time is also depends on the resolution of the image as well. The white background number plates took less time than the green background number plates. In experiment working with different type of vehicles number plate like car, carry van and trucks and their colors are different as well get significant result. The proposed method can deal with the number plate having 0-10 degrees tilt and rotation. But need to choose the best template for the process as well, if templates are cropped or clipped then may recognize wrong character. Besides in time of experiment segmentation should be correct otherwise recognition will hampered, if $\odot$ is clipped in upper side, then it may recognize as $\sqcup$ as the proposed method looking for the best match . Comparing with other results mentioned above, the proposed method has significant accuracy and better processing time.

In short, the binarazation using Otsu method and filling with dilation and erosion produce higher accuracy of extraction. Besides, clearing the dust and choosing good templates also gave more accuracy of segmentation, besides best matching template matching was used instead of exact matching which also improved recognition rate.

\section{CONCLUSION}

In this paper, worked with still image captured from road side vehicles and proposed a method to detect and recognize Bangla vehicle number Plate. The proposed method have greater accuracy to localize the number plate as it can detect the number plate from both side front and rear. This method can detect different background color number plates (green and white) captured in morning and evening sunlight. The method achieve a good processing time as well, take $0.18 \mathrm{sec}$ for overall process. Performance of the method increased with the quality of the image as well. For future work to improve the performance of number plate detection, can work with real time detection from videos by improving rotation correction and tilt correction of number plates, besides detection in rough weather. Template matching may face some problem to detect ambiguous characters like $\forall$ and $\checkmark$, $\odot$ and $৬$. Instead of template matching neural network, Support vector machine may use for higher accuracy to overcome this problem.

\section{REFERENCES}

[1] A. C. Roy, M. K. Hossen, D. Nag" License Plate Detection and Character Recognition System for Commercial Vehicles based on Morphological Approach and Template Matching”. 3rd International Conference on Electrical Engineering and Information Communication Technology (ICEEICT).

[2] Dipti Jadhav, ManojSabnis," Open Road Tolling in India by Pattern Recognition". International Conference on Technologies for Sustainable Development (ICTSD2015).

[3] J. M. N. D. B. Jayasekara, W. G. C. W. Kumara," Text Extraction for Sri Lankan Number Plates". 2015 8th International Conference on Ubi-Media Computing (UMEDIA): 089 - 091

[4] NaumanSaleem, H.M.Tahir, Hassam Muazzam, Umar Farooq," Automatic License Plate Recognition Using Extracted Features". 2016 4th International Symposium on Computational and Business Intelligence

[5] M.M.A. Joarder, K. Mahmud, T. Ahmed, M. Kawser, and B. Ahamed, "Bangla automatic number plate recognition system using artificial neural network," Asian Trans. on Science \& Technology, vol. 02, no.01, March 2012.

[6] S. Ozbay, and E. Ercelebi, "Automatic vehicle identification by plate recognition," in Proc. of Word Academy of Science Engineering and Technology, vol. 09, no. 04, pp. 222-225, November 2005.

[7] A. Abd El Rahman, A. Hamdy and F.Zaki," Automatic Arabic Number Plate Recognition". 2013 Second International Japan-Egypt Conference on Electronics, Communications and Computers (JEC-ECC)

[8] A. H. Ashtari, M. J. Nordin, and S. M. M. Kahaki, “A New Reliable Approach for Persian License Plate Detection on Colour Images," in Proc. International Conf. on Electrical Engineering and Informatics (ICEEI), Bandung, Indonesia, pp. 1-5, 2011.

[9] F. Faradji, A. H. Rezaie, and M. Ziaratban, "A Morphological-Based License Plate Location," ICIP07,pp. 57-60, 2007.

[10] Naito, T., Tsukada, T., Yamada, K., Kozuka, K., and Yamamoto, S.," Robust License-Plate Recognition Method for Passing Vehicles under Outside Environment", IEEE transactions on vehicular technology, vol.49, 2000.

[11] Qadri, M. T., and Asif, M., "Automatic Number Plate Recognition System for Vehicle Identification using 
Optical Character Recognition", IEEE International Conference on Education Technology and Computer, pp.335-338, 2009.

[12] Zhang, S., Zhang, M., and Ye, X., "Car Plate Character Extraction Under Complicated Environment," IEEE Int. Conf. Syst. Man Cybern., vol..5, pp.4722-4726, 2004.

[13] R. de Queiroz, Z. Fan, and T. Tran, "Optimizing BlockThresholding Segmentation for compound images", IEEE Transactions On Image Processing, vol.9, no.9, September 2000, pp. 1461-1471.

[14] D. Zhou, Y. Liu, and X Cai, "An efficient and robust corner detection algorithm", Proceedings of the 5th World Congress on Intelligent Control and Automation, Hangzhou: Press of Zhejiang University, 2004, pp. 4020-4024.

[15] Zhigang $\mathrm{Xu}$, Honglei Zhu, An Efficient Method of Locating Vehicle License Plate. Third International Conference on Natural Computation (ICNC 2007) 07695-2875-9/07

[16] Vinh Du Mai,DuoqianMiao,Ruizhiwang," Building a license plate recognition system for Vietnam tollbooth". Proceedings of the Third Symposium on Information and communication technology, pages 107-114.

[17] Liu P, Li G, Tu D, et al. Low-quality License Plate Character Recognition Based on CNN[C]. International symposium on computational intelligence and design, 2015: 53-58.

[18] Liu D Y, Song H, Pan Q. "License Plate Recognition Based on Neural Network Algorithm to Improve Research". Advanced Materials Research, 2013, 860863:2892-2897.

[19] Feng J, Li Y, Chen M. "The research of vehicle license plate character recognition method based on artificial neural network".International Asia Conference on Informatics in Control, Automation and Robotics. IEEE, 2010:317-320.

[20] Y. Yanamura, M. Goto, D. Nishiyama, M. Soga, Ht. Nakatani and H. Saji, "Extraction and Tracking of the License Plate using Hough Transform and Voted Block Matching," In proceedings of IEEE Intelligent Vehicles Symposium, pp. 243-246, 2003.

[21] C.Nelson Kennedy Babu, Krishnan Nallaperumal,, "A License Plate Localization using Morphology and Recognition”. 2008 Annual IEEE India Conference.

[22] Wen, Y., Lu, Y., Yan, J.Q., Zhou, Z.Y., Karen, M., Deneen, V., Shi, P.F. 2011. An Algorithm for License Plate Recognition Applied to Intelligent Transportation System.IEEE. Trans. Intel.Trans.Systems. 12, 3, 830845 